\title{
Structural synthesis of parallel manipulators
}

\author{
Rasim Alizade *, Çăğdaş Bayram \\ Mechanical Engineering Department, Izmir Institute of Technology, Urla 35437, Izmir, Turkey \\ Received 5 October 2002; received in revised form 20 January 2004; accepted 24 February 2004
}

\begin{abstract}
In this paper, structural synthesis and classification of parallel manipulators are considered. Parallel manipulators are classified according to their platform type(s) and connections between them. New and revised methods and formulations for designing variety of parallel manipulators with single and multiple platforms are presented along with examples.

(C) 2004 Elsevier Ltd. All rights reserved.
\end{abstract}

\section{Peзюne}

В представленной работе рассматриваются вопросы структурного синтеза и классификация манипуляторов параллельной структурьг. Классификация пространственньгх механизмов параллельной структуры основан на методе развития платформ и связей между ними. Новые и перессмотренньг методы и формулировки по проектированию манипуляторов параллельной структуры иллюстрируются различньги примерами.

(C) 2004 Elsevier Ltd. All rights reserved.

\section{Introduction}

Machine science is always in development and subjects like robotics, mechatronics, micromechanisms, etc. are evolving rapidly today, in the beginning of XXI century. The accumulation of scientific investigations and increased manufacturing activity in these areas inevitably arise the necessity to review the older theory. A new theory should be created in the new direction and be more suitable for use in practice.

\footnotetext{
* Corresponding author. Tel.: +90-232-7506544; fax: +90-232-7506505.

E-mail address: rasimalizade@iyte.edu.tr (R. Alizade).
} 
One of the most important steps in designing a robotic mechanical system is to solve the problem of structural synthesis of mechanisms. An inefficient design for a mechanism, from structural point of view, will lead to excessive loads at kinematic pairs. At this point, it is possible to refine and correct the manipulator or robot structure with the help of structural synthesis.

In year 1883 Grübler [1,2] described a structural formula for planar mechanisms for a range of functional determinant $(\lambda=3, \lambda=2)$ and kinematic chains with revolute, cam and prismatic pairs, and another equation for only prismatic pairs. In 1887, Somov [3] described a structural formula for spatial and planar mechanisms $(\lambda=6, \lambda=3)$. Many other scientists devoted their studies in this direction as, P.L. Chebyshev, D. Silvester, K.I. Gokhman, R. Muller, A.P. Malushev, F. Wittenbayer, K. Kutzbah, V.V. Dobrovolskyi, J.F. Moroshkin, B. Paul, K.H. Hunt, N. Boden, O.G. Ozol and so on.

Further development of structural formulas to find degree of freedom (dof) of complex mechanisms with variable general constraint was done by Freudenstein and Alizade [4]. This formulation incorporated various magnitudes of constraints imposing linear loop closures considering geometrical connections of kinematic pairs and also independent variables of relative displacements. In 1988, the first author [5] presented a new structural formula in which; mobile platform types, number of mobile platforms, number of connections between en platforms and so were included along with mobility of kinematic pairs. Analysis of physical essence and geometrical interpretation of various constraint parameters were also given [6,7].

A structural group can be defined as a mechanism or manipulator having zero degree of freedom. A simple structural group is a structural group which cannot be split into other structural groups. Defining simple structural groups and constructing mechanisms using their combinations was studied, striving to systemize the methods for investigating the mechanisms. In year 1916, Assur [8] introduced the formal structural classification for planar mechanisms and in 1936, Artobolevski [9] introduced the structural classification for both planar and spatial mechanisms using the loop development method. The method of Dobrovolskyi [10] was based on the principle of dividing joints but the structural synthesis made by Kojevnikov [11] was using the method of developing joints. Structural synthesis by Ozol [12] was based on mechanisms topological property.

The methods reported by Freudenstein [13], Dobrjanskyi and Freudenstein [14] and Davies and Crossley [15] were based on graph theory. Sohn and Freudenstein [16] used the concept of dual graphs and generated kinematic chains with up to 11 links and 2 dof. A computer-aided method for generating planar kinematic chains was also introduced [17]. Professor Hunt and coworkers [18] presented the method for generating the chains using a test for avoiding isomorphism. The method presented in [19] is based on the concept of loop formation, which cancels the necessity of the test for isomorphism.

The 6-dof parallel manipulator introduced by Stewart [20] took great interest. Further development on structural synthesis of spatial mechanisms [21], and new structural classification of mechanisms [22] was given, using the method of developing basic links (platforms) and their connections.

Our method of structural synthesis of parallel manipulators begins with determining the simple structural groups for a given set of parameters of synthesis. Then a number of required actuators are added to the group to form the manipulator. Other aspects regarding manipulator design such as geometry and kinematics are also examined from structural point of view. 


\section{Structural formula}

In this section, a structural formula that is a function of number of branches, platforms and the sum of mobility of kinematic pairs for a parallel manipulator is described.

A platform is an $m$-pair link where $m \geqslant 3$. By definition, two platforms may not form joints with each other in the same manipulator. A parallel manipulator has at least one mobile platform. A mobile platform is connected to the frame (motionless link/fixed link) via kinematic chains that are generally referred as legs or limbs. In the case of a manipulator having more then one mobile platforms, these mobile platforms may be connected to each other by means of kinematic chains which we will refer as branches. A leg or a branch is a serial kinematic chain.

Consider a parallel manipulator including at least two mobile platforms. Let $B, j_{\mathrm{p}}, c, c_{\mathrm{b}}, c_{1}$ denote the number of mobile platforms, total number of joints on the platforms, total number of connections (that is sum of number of legs and branches), total number of branches and total number of legs of the manipulator respectively. By definition it follows that:

$$
c=c_{\mathrm{b}}+c_{1}
$$

Assume that each leg or branch in the manipulator consists of only one link. The total number of moving links would then be:

$$
n=B+c_{\mathrm{b}}+c_{1}
$$

where $n$ is the total number of moving links in the manipulator.

For the general case, a leg or branch may consist of more than one link. Let a leg consists of $n_{1}=1+\tilde{n}_{1}$ links where, $\tilde{n}_{1}$ is the number of additional links. In this case, there should also be $\tilde{j}_{1}$ number of additional joints on the limb to connect the additional links such that $\tilde{j}_{1}=\tilde{n}_{1}$. The same is true for a branch which can be expressed by $\tilde{j}_{\mathrm{b}}=\tilde{n}_{\mathrm{b}}$ where $\tilde{j}_{\mathrm{b}}, \tilde{n}_{\mathrm{b}}$ are the number of additional joints and links on a branch. Eq. (2) is extended as:

$$
n=B+c_{\mathrm{b}}+c_{1}+\sum_{i=1}^{c_{1}} \tilde{n}_{1, i}+\sum_{i=1}^{c_{\mathrm{b}}} \tilde{n}_{\mathrm{b}, i}
$$

Likewise, assume that each leg or branch in a structural group consists of only one link. So that each branch is formed by connecting two joints on different mobile platforms with one link and each leg is formed by connecting a joint on a mobile platform to a joint on the frame with a link. Thus, the total number of joints in the structural group would be:

$$
j=j_{\mathrm{p}}+c_{1}
$$

For the general case, as explained above, Eq. (4) is extended as:

$$
j=j_{\mathrm{p}}+c_{1}+\sum_{i=1}^{c_{1}} \tilde{j}_{1, i}+\sum_{i=1}^{c_{\mathrm{b}}} \tilde{j}_{\mathrm{b}, i}
$$

Euler's formula to find the number of independent loops in a closed kinematic chain is stated as follows:

$$
L=j-n
$$


where $L$ is the number of independent loops, $j$ is the number of joints and $n$ is the number of moving links in the mechanism.

Substituting Eqs. (3) and (5) in (6), we get the formula that gives the number of independent loops as:

$$
L=j_{\mathrm{p}}-B-c_{\mathrm{b}}
$$

Since a leg is connected to only one joint on a mobile platform and a branch is connected to two joints on different mobile platforms, the total number of joints on the mobile platforms in a parallel manipulator can be described as:

$$
j_{\mathrm{p}}=2 c_{\mathrm{b}}+c_{1}
$$

and using Eq. (1) in Eq. (8)

$$
j_{\mathrm{p}}=c_{\mathrm{b}}+c
$$

Substituting Eq. (9) into Eq. (7) we obtain:

$$
L=c-B
$$

In a previous study by Freudenstein and Alizade [4], an equation to find the degree of freedom of mechanisms was given as:

$$
W=\sum_{i=1}^{j} f_{i}-\lambda L
$$

where $W$ is the degree of freedom of the mechanism, $j$ is the total number of joints in the mechanism, $f_{i}$ is the mobility of the $i$ th joint, $L$ is the number of independent closed loops and $\lambda$ is the degree of freedom of an unconstrained rigid body moving in the workspace of the mechanism.

Substituting Eq. (7) in Eq. (11) we get:

$$
W=\sum_{i=1}^{j} f_{i}-\lambda\left(j_{\mathrm{p}}-B-c_{\mathrm{b}}\right)
$$

Likewise, substituting Eq. (10) in Eq. (11) we get:

$$
W=\sum_{i=1}^{j} f_{i}-\lambda(c-B)
$$

Finally, to obtain the equation for structural groups we set $W=0$ in Eqs. (12) and (13) to get:

$$
\begin{aligned}
& \sum_{i=1}^{j} f_{i}=\lambda\left(j_{\mathrm{p}}-B-c_{\mathrm{b}}\right) \\
& \sum_{i=1}^{j} f_{i}=\lambda(c-B)
\end{aligned}
$$

Eqs. (14) and (15) describe structural groups with at least one mobile platform. 


\section{Structural synthesis of parallel manipulators}

A parallel manipulator with a certain degree of freedom can be obtained by taking an appropriate structural group and adding the required number of actuators. So, our starting point will be Eq. (15). For the sake of clarity, Eq. (15) is rewritten as:

$$
f_{\mathrm{t}}=\lambda(c-B)
$$

where $f_{\mathrm{t}}$ is the sum of mobility of all joints in the structural group, $f_{\mathrm{t}}=\sum_{i=1}^{j} f_{i}$.

From now on in this paper, the term joint will be used in account for one mobility kinematic pair unless otherwise specified.

The proposed method for structural synthesis starts with selecting the number of mobile platforms $B$ and total number of joints on platforms $j_{\mathrm{p}}$. The first step is to find the number of possible different structural groups using these parameters. To form a structural group composed of mobile platforms, branches and legs, the necessary conditions are:

(a) Each joint on a mobile platform has to be connected either by a branch to a joint on another mobile platform or by a leg to the frame.

(b) There should be at least one branch connecting each mobile platform to another mobile platform.

(c) There should be at least two legs connecting mobile platforms to the frame.

To minimally satisfy condition (b), there should be at least $c_{\mathrm{b}}=B-1$ branches. In this configuration, we have a total of $j_{\mathrm{p}}-2(B-1)$ unconnected joints left on the mobile platforms. When these free joints are connected to the frame to satisfy condition (a), we have $c_{1}=j_{\mathrm{p}}-2(B-1)$ legs. So in this case, we find the maximum total number of branches and legs that can be used in a structural group, given as:

$$
\begin{aligned}
c_{\max } & =c_{\mathrm{b}, \min }+c_{1, \max }=[B-1]+\left[j_{\mathrm{p}}-2(B-1)\right] \\
c_{\max } & =1+j_{\mathrm{p}}-B
\end{aligned}
$$

To minimally satisfy condition (c), $c_{1}=2$. In this case we have $j_{\mathrm{p}}-2$ joints on the platform left to connect branches thus we will have $c_{\mathrm{b}}=0.5\left(j_{\mathrm{p}}-2\right)=0.5 j_{\mathrm{p}}-1$. So in this case, we find the minimum number of branches that can be used in a structural group, given as:

$$
\begin{aligned}
& c_{\text {min }}=c_{\mathrm{b}, \text { max }}+c_{1, \text { min }}=0.5 j_{\mathrm{p}}-1+2 \\
& c_{\text {min }}=1+0.5 j_{\mathrm{p}}
\end{aligned}
$$

In the case of $j_{\mathrm{p}}$ being and odd number, the value of $c_{\min }$ is rounded up. Finally, using Eqs. (17) and (18) we may state that $c_{\min } \leqslant c \leqslant c_{\max }$ which, can also be written as:

$$
1+0.5 j_{\mathrm{p}} \leqslant c \leqslant 1+j_{\mathrm{p}}-B
$$

From Eq. (15), it is clear that the number of different structural groups for a given $B$ depends on the number of different values that $c$ can take. Since $c$ is a natural number existing in a closed interval $\left[c_{\min }, c_{\max }\right]$, the number of different values that $c$ may take is given by $c_{\max }-c_{\min }+1$ that is:

$$
G=0.5 j_{\mathrm{p}}-B+1
$$


where $G$ is the number of different values that $c$ may take which is also the number of different structural groups that may be generated with a given $B$ and $j_{\mathrm{p}}$. In the case of $j_{\mathrm{p}}$ being and odd number, the value of $G$ is rounded down.

The procedure can be summarized step by step as follows:

(1) Select values for $B$ and $j_{\mathrm{p}}$.

(2) The number of different structural groups is given by Eq. (20).

(3) Select a value for $c$, in the interval given by Eq. (19).

(4) Calculate the number of branches from Eq. (9).

(5) Calculate the number of legs from Eq. (1).

(6) Calculate $f_{\mathrm{t}}$ from Eq. (15).

(7) Place the joints on branches and legs.

(8) Decide on the place to add the actuators. The dof of the manipulator is equal to the number of actuators added. Note that one may place the actuators on legs or branches and also more than one actuator may be placed on the same leg or branch.

(9) Using the principle of interchangeability of kinematic pairs, replace the single mobility kinematic pairs with other kinematic pairs as desired.

Example 1. A spatial parallel manipulator with four degrees of freedom is required, we want to use two triangular platforms. (1) $B=2, j_{\mathrm{p}}=3+3=6, W=4, \lambda=6$. (2) Eq. (20): $G=0.5 \times 6-2+1=2$. Since we have only two different structural groups, we decided to find both of them at once. (3) Eq. (19): $c=4$ and also $c=5$. (4 and 5) For $c=4 \rightarrow c_{\mathrm{b}}=2, c_{1}=2$, for $c=5 \rightarrow c_{\mathrm{b}}=1, c_{1}=4$. (6) Eq. (16): for $c=4 \rightarrow f_{\mathrm{t}}=6(4-2)=12$, since we have a total of four branches and legs, it is convenient to place $12 / 4=3$ joints on each leg or branch. For $c=5 \rightarrow f_{\mathrm{t}}=6(5-2)=18$, since we have a total of five branches and limbs, it is convenient to place three joints on each leg or branch and place the remaining three joints on some of the branches or legs as we like. (7) The placement of joint for $c=4$ and 5 is given in Fig. $1 \mathrm{~b}$ and c respectively (8 and 9). In Fig. 1b, two actuators are placed on branches and two actuators are placed on legs. In Fig. 1c, all actuators are placed on the legs. Therefore the synthesis is concluded.

In general, the parallel manipulators are designed such that all actuators rest on the frame. So that the manipulator does not carry the weight of its actuators, resulting in lower inertia and actuation torque/force requirements. This means that all actuators are placed in legs and in each leg only one actuator is placed. To synthesize such a manipulator, $c_{1}$ is set equal to $W$. Second step of the procedure is skipped, $c_{\mathrm{b}}$ is calculated directly from Eq. (8). In third step, $c$ is calculated directly from Eq. (1). The rest of the procedure is the same. Note that only one such structural group exist for a given $j_{\mathrm{p}}$ and $B$.

Example 2. Determine the structure of a multi-platform spatial parallel manipulator with six degree of freedom $(W=6)$. The six input actuators will be located on the frame. We will take one quadrilateral and two hexagonal platforms, thus $B=3$. Now, we may find $j_{\mathrm{p}}$ by summing the number of joints in each platform as $j_{\mathrm{p}}=4+6+6=16$. We need six legs since we need to put six actuators $\left(c_{1}=W\right)$, so we take $c_{1}=6$. From (8), we find the number of branches as 


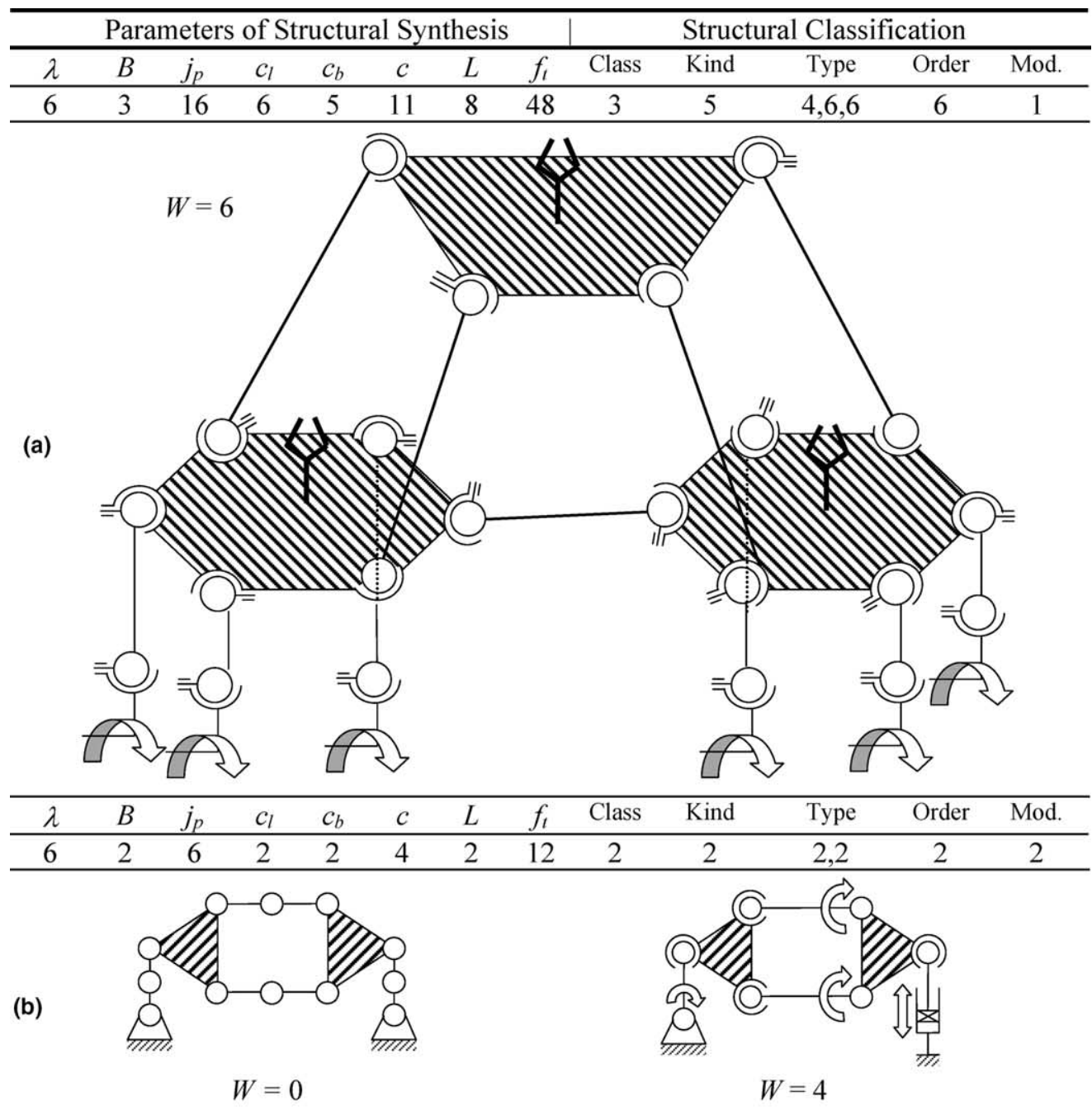

\begin{tabular}{ccccccccccccc}
\hline$\lambda$ & $B$ & $j_{p}$ & $c_{l}$ & $c_{b}$ & $c$ & $L$ & $f_{t}$ & Class & Kind & Type & Order & Mod. \\
\hline 6 & 2 & 6 & 4 & 1 & 5 & 3 & 18 & 2 & 1 & 2,2 & 4 & 2 \\
\hline & & & & & & & & & & & &
\end{tabular}

Fig. 1. Structural synthesis and classification of various parallel manipulators. 
$c_{\mathrm{b}}=(16-6) / 2=5$. The total number of branches and legs is then $c=11$, the number of independent loops is $L=8$, total number of mobility of kinematic pairs as $f_{\mathrm{t}}=48$. Using only single mobility kinematic pairs, the number of joints on each branch or leg is $48 / 11$, let us denote this as $j_{\mathrm{c}}=4(4)$, the latter 4 being the remainder. We will put five joints on four branches and four joints on the remaining six legs and one branch. Note that for this particular example, we have only one way to place the remaining joints since we cannot put more than five joints on a single branch. After adding the actuators and substituting the joints using principle of interchangeability, our task is concluded. One such manipulator is given in Fig. 1a.

At this point we can give the following definitions for structural classification:

Class of a structural group is the number of mobile platforms it has.

Type of a structural group is expressed as a set of numbers such that each number in the set is the number of joints on one of the mobile platforms.

Kind of a structural group is the number of branches it has.

Order of a structural group is the number of its legs.

Modification: Based on the last step of structural synthesis procedure. If all the joints in the structural group are revolute joints or interchangeable with revolute joints like universal joint $(\mathrm{U} \rightarrow \mathrm{RR}$ ) or spherical joint ( $\rightarrow \mathrm{RRR}$ ), then it is called as first modification. If the structural group contains at least one prismatic joint or a cylindrical joint $(C \rightarrow R P)$ and possibly $R, U$ or $\mathrm{S}$ joints, then it is called as second modification. If a structural group contains at least one screw joint and possibly R, U, S, P or C joints, then it is called as third modification.

A sample classification of various parallel manipulators is given in Fig. 1.

\section{Geometrical structural synthesis of parallel manipulators}

In this section, we will focus on three subjects as: (1) Investigation of possible serial branch and leg configurations. (2) Creation of complex branch or leg structure by combining serial kinematic chains and structural groups. (3) Multi-module or modular parallel manipulators (Fig. 2).

\subsection{Simple branch and leg configurations}

A leg or a branch is previously defined as a serial kinematic chain. As an example, a leg consisting of three revolute joints $(\mathrm{R}-\mathrm{R}-\mathrm{R})$ can be replaced by a universal and a revolute joint (U-R). On the base of interchangeability of kinematic pairs, considering only lower kinematic pairs, a $\left(\mathrm{p}_{1}-\mathrm{p}_{1}\right)$ chain may be replaced by a $\left(\mathrm{p}_{2}\right)$ joint or a $\left(\mathrm{p}_{1}-\mathrm{p}_{1}-\mathrm{p}_{1}\right)$ chain may be replaced by a $\left(\mathrm{p}_{3}\right)$ joint and vice versa where $\mathrm{p}_{n}$ represents an $n$ mobility kinematic pair, $1 \leqslant n \leqslant 3$. By writing a simple computer code, one may find the number of possible branch and leg configurations for branches and legs having various degrees of freedom.

This information is summarized in Table 1. For example, the last column gives information on a chain having 2 dof (row 1). So the number of joints can only be 2 (row 2) as $\mathrm{p}_{1}-\mathrm{p}_{1}$ (last row). There are four possible configurations (row 3) as $\mathrm{R}-\mathrm{R}, \mathrm{P}-\mathrm{P}, \mathrm{R}-\mathrm{P}$ and $\mathrm{P}-\mathrm{R}$ but since 
(a)

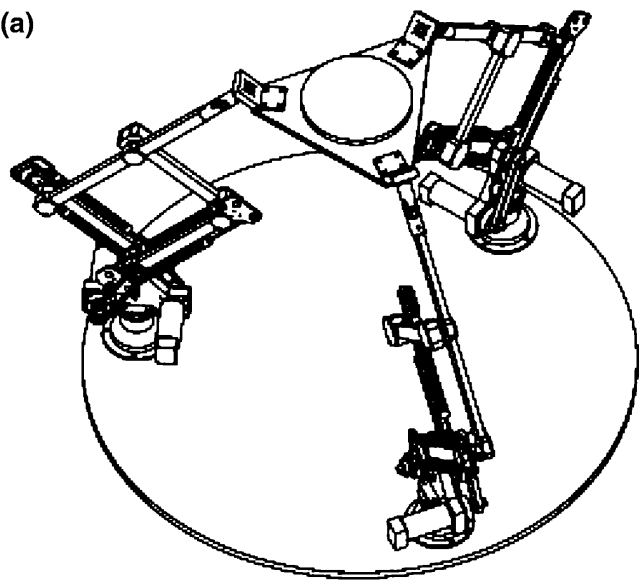

(b)

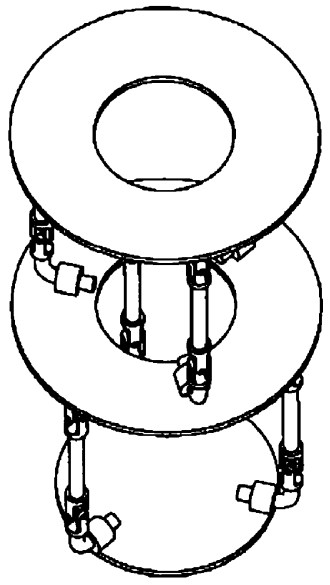

(c)

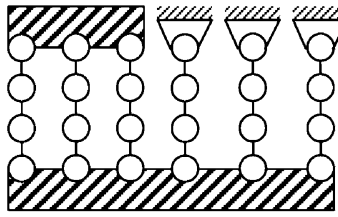

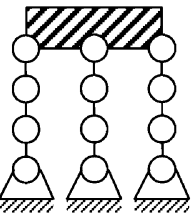

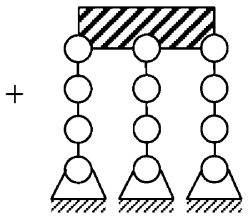

Fig. 2. (a) 6-dof spatial parallel manipulator with a triangular platform. (b) $2 \times 3$-dof modular spatial parallel manipulator. (c) Formation of a structural group using two simple structural groups.

Table 1

Number of possible branch or leg structures with $2 . .6$ dof

\begin{tabular}{|c|c|c|c|c|c|c|c|c|c|c|c|c|c|c|}
\hline \multirow{2}{*}{$\begin{array}{l}\text { Number of } \\
\text { joints in the branch or leg }\end{array}$} & \multicolumn{5}{|c|}{$\begin{array}{l}\text { Branch or leg dof } \\
6\end{array}$} & \multicolumn{4}{|l|}{5} & \multicolumn{3}{|l|}{4} & \multirow{2}{*}{$\frac{3}{3}$} & \multirow{2}{*}{$\begin{array}{l}2 \\
2\end{array}$} \\
\hline & 2 & 3 & 4 & 5 & 6 & 2 & 3 & 4 & 5 & 2 & 3 & 4 & & \\
\hline $\begin{array}{l}\text { Number of } \\
\text { all possible configurations }\end{array}$ & 1 & 32 & 122 & 130 & 42 & 4 & 24 & 62 & 26 & 8 & 25 & 15 & 8 & 4 \\
\hline $\begin{array}{l}\text { Number of } \\
\text { repeated configurations }\end{array}$ & 0 & 14 & 58 & 62 & 19 & 2 & 10 & 31 & 10 & 3 & 11 & 5 & 2 & 1 \\
\hline $\begin{array}{l}\text { Number of } \\
\text { unique configurations }\end{array}$ & 1 & 18 & 64 & 68 & 23 & 2 & 14 & 31 & 16 & 5 & 14 & 10 & 6 & 3 \\
\hline \multirow[t]{10}{*}{ Structural representation } & $\mathrm{p}_{3}-\mathrm{p}_{3}$ & $\mathrm{p}_{1-}$ & $\mathrm{p}_{1-}$ & $\mathrm{p}_{1-}$ & $\mathrm{p}_{1}-$ & $\mathrm{p}_{3}-$ & $\mathrm{p}_{1}-$ & $\mathrm{p}_{1}-$ & $\mathrm{p}_{1-}$ & $\mathrm{p}_{1}-$ & $\mathrm{p}_{2}-$ & $\mathrm{p}_{1}-$ & $\mathrm{p}_{1}-$ & $\mathrm{p}_{1}-$ \\
\hline & & $\mathrm{p}_{2}-$ & $\mathrm{p}_{1}-$ & $\mathrm{p}_{1}-$ & $\mathrm{p}_{1}-$ & $\mathrm{p}_{2}$ & $\mathrm{p}_{2}-$ & $\mathrm{p}_{1}-$ & $\mathrm{p}_{1}-$ & $\mathrm{p}_{3}$ & $\mathrm{p}_{1}-$ & $\mathrm{p}_{1}-$ & $\mathrm{p}_{1}-$ & $\mathrm{p}_{1}$ \\
\hline & & $\mathrm{p}_{3}$ & $\mathrm{p}_{1}-$ & $\mathrm{p}_{1-}$ & $\mathrm{p}_{1}-$ & & $\mathrm{p}_{2}$ & $\mathrm{p}_{1-}$ & $\mathrm{p}_{1-}$ & & $\mathrm{p}_{1}$ & $\mathrm{p}_{1}-$ & $\mathrm{p}_{1}$ & \\
\hline & & & $\mathrm{p}_{3}$ & $\mathrm{p}_{1-}$ & $\mathrm{p}_{1}-$ & & & $\mathrm{p}_{2}$ & $\mathrm{p}_{1}-$ & & & $\mathrm{p}_{1}$ & & \\
\hline & & & & $\mathrm{p}_{2}$ & $\mathrm{p}_{1}-$ & & & & $\mathrm{p}_{1}$ & & & & & \\
\hline & & & & & $\mathrm{p}_{1}$ & & & & & & & & & \\
\hline & & $\mathrm{p}_{2}-$ & $\mathrm{p}_{1}-$ & & & & & & & $\mathrm{p}_{2}-$ & & & & \\
\hline & & $\mathrm{p}_{2}-$ & $\mathrm{p}_{1}-$ & & & & & & & $\mathrm{p}_{2}$ & & & & \\
\hline & & $\mathrm{p}_{2}$ & $\mathrm{p}_{2}-$ & & & & & & & & & & & \\
\hline & & & $\mathrm{p}_{2}$ & & & & & & & & & & & \\
\hline
\end{tabular}


$\mathrm{R}-\mathrm{P}$ and $\mathrm{P}-\mathrm{R}$ are the same from structural point of view, (row 4) we have $4-1=3$ unique configurations (row 5).

\subsection{Complex branch and leg configurations}

In this paper, structural synthesis of parallel manipulators consisting of serial branch or leg structures is considered. However, a leg or branch can also be a combination of serial kinematic chains and simple structural groups such as one shown in Fig. 2a. Each of three branches has two actuators. To connect the actuators to the platform, an $\mathrm{R}-\mathrm{R}-\mathrm{R}$ planar structural group is added to each branch. These types of manipulator structures also exist in the literature but their structure is created more or less intuitively. A methodology to describe these structures is considered as a future work.

\subsection{Modular parallel manipulator}

Let us synthesize a structural group, using the procedure presented in Section 3 , with $j_{\mathrm{p}}=9$, $B=2$, type 3-6. One of the possible structural groups will have $c_{1}=3, c_{\mathrm{b}}=3, f_{\mathrm{t}}=24$. This structural group is illustrated in Fig. 2c. However, this structural group is not simple since it can be split into two identical simple structural groups with $B=1, j_{\mathrm{p}}=3, c_{1}=3, c_{\mathrm{b}}=0, f_{\mathrm{t}}=12$ as shown in Fig. 2c.

Using non-simple structural groups, we can construct multi-module or modular parallel manipulators. However, it is important to identify these non-simple structural groups since they are not unique structural groups such that they can be formed by combining two or more simple structural groups. A 6-dof spatial multi-module parallel manipulator is given in Fig. 2b. Another interesting multi-module parallel manipulator consisting of spherical parallel simple structural groups was given in [23].

\section{Kinematical structural synthesis of parallel manipulators}

In the previous sections, a methodology to describe the structure of branches and legs was described. In this section the authors wishes to present guidelines for:

(1) Selection of construction parameters of a branch or leg.

(2) Inverse kinematics and leg configurations.

(3) Over constrained parallel manipulators.

\subsection{Selection of construction parameters of a leg or branch}

The branches and legs of a parallel manipulator may be considered as separate serial kinematic chains with $W_{\mathrm{c}}=2 . .6$, where $W_{\mathrm{c}}$ is the dof of the chain. Consider kinematical structural synthesis of a branch with three revolute joints, the task is to find number of possible structural schemes and construction parameters of the branch. The structural scheme of a kinematic chain can be described with unit screws placed along the links and axes of kinematic pairs. In Fig. 3, the 


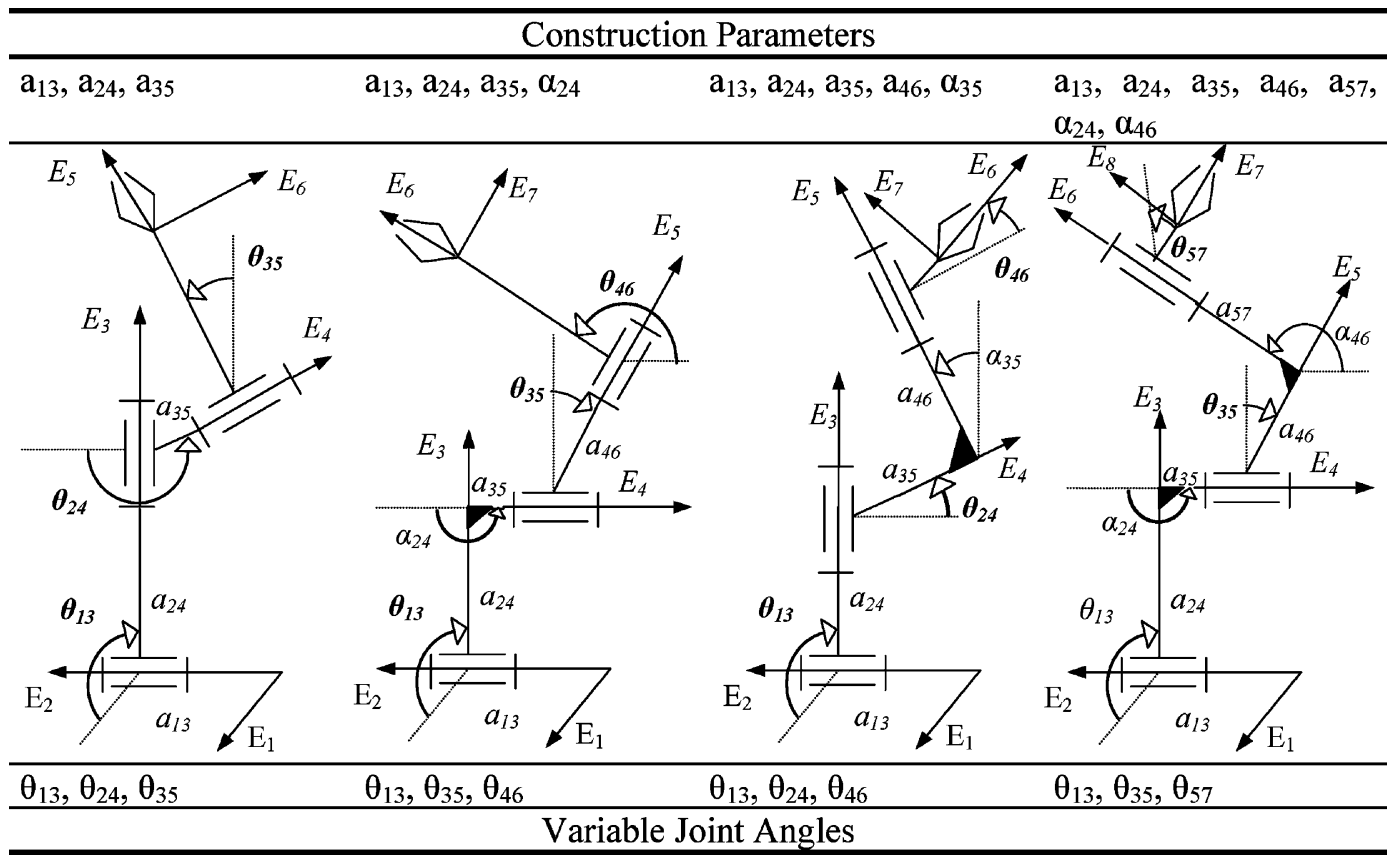

Fig. 3. Geometrical structural synthesis of an $\mathrm{R}-\mathrm{R}-\mathrm{R}$ kinematic chain.

number of unit screws describing the branches is $6,6,7$ and 8 from left to right respectively. There are four combinations of variables and construction parameters. The combination of revolute and prismatic pairs for this kind of branch equals 8 . Theoretically there are $8 \times 4=32$ structural schemes with different variables and construction parameters. For branch with four joints we have eight combinations and $15 \times 8=120$ different theoretical schemes. For branch with five joints we have 14 combinations produce $26 \times 14=364$ structural schemes.

\subsection{Inverse kinematics and leg configuration}

The spatial parallel manipulator given in Fig. 4a has 6 dof, actuated by three linear and three rotary actuators. Each leg is a serial 6-dof U-P-S kinematic chain. Inverse kinematic solution is simple since we can find the position of the center of spherical joints lying on the platform given the platform location, and then calculate the leg lengths and rotary actuator angles and so on easily (Fig. 4a).

It was shown that [24], for spatial parallel manipulators with single mobile platform, the workspace of the manipulators increases and the inverse kinematic analysis becomes easier to solve if dof of one leg of the manipulator is selected as equal to the dof of the manipulator as given in Fig. 4b-d.

Consider the 5-dof manipulator given in Fig. 4b. It has one platform with five joints, $4 \times \mathrm{U}-\mathrm{P}-\mathrm{S}$ and $1 \times \mathrm{U}-\mathrm{P}-\mathrm{U}$ leg. Taken individually, four legs has 6 dof and the remaining leg has 5 dof. The platform location is defined by five independent parameters in space. For the inverse position analysis assume that we know all three independent parameters describing the platform 
(a)
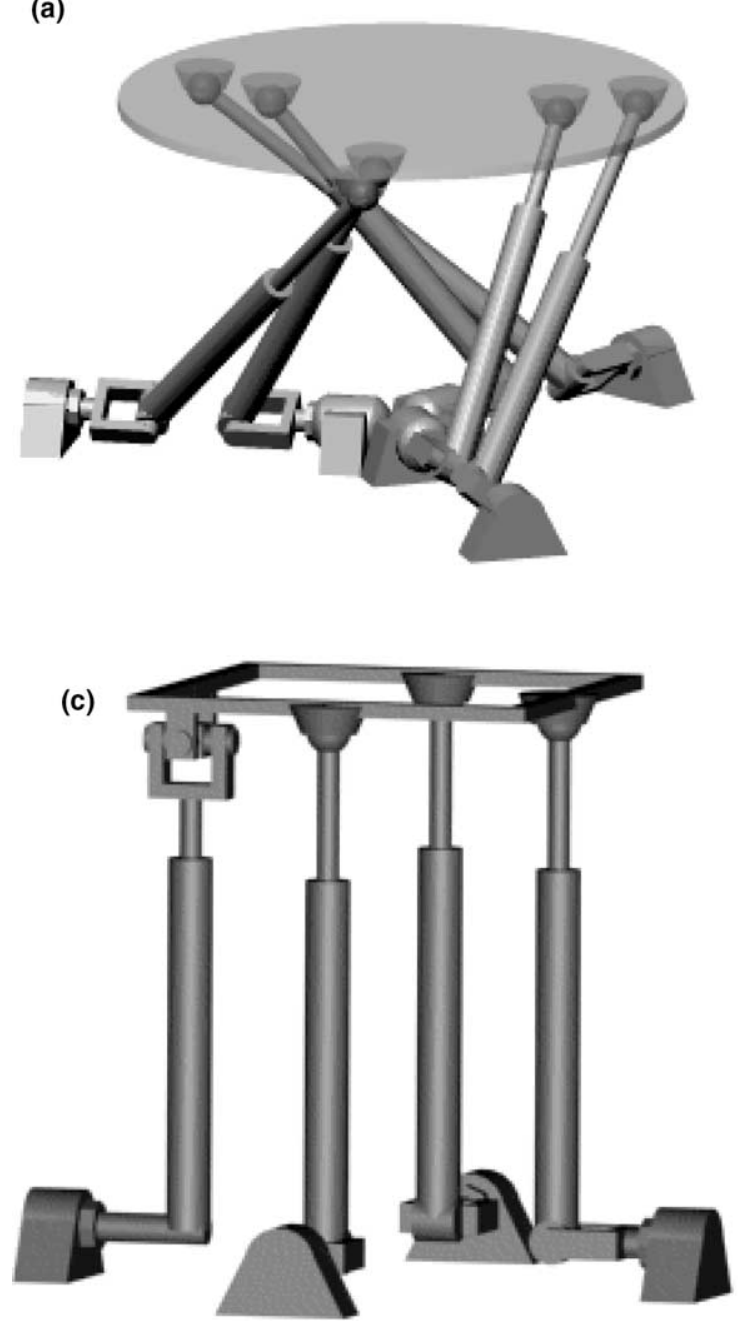

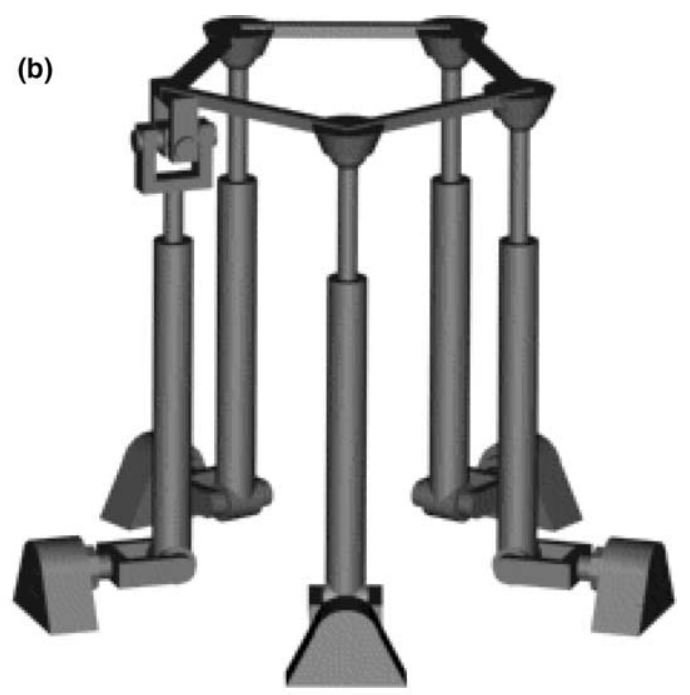

(d)

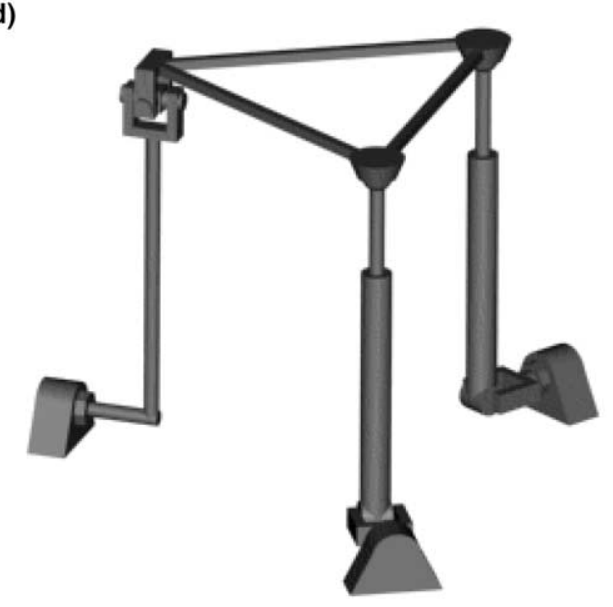

Fig. 4. Spatial parallel manipulators with (a) 6 dof (b) 5 dof (c) 4 dof and (d) 3 dof.

orientation and two independent parameters describing the platform position. Firstly, all joint variables of the $\mathrm{U}-\mathrm{P}-\mathrm{U}$ leg is solved. Now that it is possible to find the third dependent parameter describing the position of the platform, by making the direct position analysis of the U-P-U leg as a serial manipulator. Finally, we can find the joint variables of the remaining legs U-P-S legs since we found six parameters describing the position and the orientation of the platform completely.

For the 4-dof and 3-dof parallel manipulators given in Fig. 4c and d respectively, one can use the same approach. Firstly, solve the inverse position analysis of the leg with lower dof. Secondly, solve the direct position analysis of that leg to find the six parameters completely defining the position and orientation of the platform. Finally, solve the inverse kinematics of the remaining legs. 


\subsection{Over constrained parallel manipulators}

Other than spatial parallel manipulators, a parallel manipulator may also operate in a subspace. These type of manipulator and mechanisms are referred as over constrained manipulator or mechanisms. The two best known subspaces are planar and spherical, both identified by $\lambda=3$. A planar manipulator or mechanism is identified by all its joint axes being parallel and a spherical manipulator or mechanism is identified by all its joints intersecting at a common point, these are called as angular constraints. Now we use spherical and planar parallel manipulators, spherical five or six link manipulators widely in practice. By improving the analytical methods, it can be possible solve the task of structural synthesis to find new types of manipulators with angular and/ or linear constraints.

\section{Conclusions}

A structural formula for parallel manipulators, having at least one mobile platform and working in space or in a subspace, is presented. A procedure to synthesize the structures of parallel manipulators using simple structural groups is presented. A classification for parallel manipulators based on the number of mobile platforms, number of joints on the mobile platforms, number of legs and branches and types of kinematic pairs is given along with examples. Geometrical and kinematical structural synthesis subjects are defined and general guidelines are presented with examples and tables.

\section{References}

[1] M. Grübler, Allgenmeine eigenschaften für swangläufiden ebenen kinematischen ketten, part I (29) (1883) 167-200.

[2] M. Grübler, Allgenmeine eigenschaften für swangläufiden ebenen kinematischen ketten, part II (64) (1885) 179229.

[3] P.O. Somov, On the dof of kinematic chains, J. P. Chem. 19 (9) (1887) $443-477$ (in Russian).

[4] F. Freudenstein, R. Alizade, On the degree of freedom of mechanisms with variable general constraint, in: IV World IFToMM Congress, England, 1975, pp. 51-56.

[5] R.I. Alizade, On the dof of kinematic chains, Azerb. Poly. Inst., Baku, 1988, pp. 3-14 (in Russian).

[6] R.I. Alizade, Physical substance and geometrical interpretation of variable constraint parameters of universal structural formula, Azerb. Tech. Univ. (1991) 3-12 (in Russian).

[7] R. Alizade, Sh. Aliyev, A. Temirov, I. Mamedov, Analysis of variable constraint parameters of universal structural formula, Azerb. Tech. Univ. 6 (1) (1997) 162-166 (in Russian).

[8] L.V. Assur, Investigation of plane linkage mechanisms with lower pairs from point of view of their structure and classification, AN USSR, 1939, pp. 49-66 (in Russian).

[9] I.I. Artobolevski, Proficiency structural analysis of mechanisms, structure and classification of mechanisms, AN USSR, 1939, pp. 49-66 (in Russian).

[10] V.V. Dobrovolskyi, Basic principles of rational classification, AN USSR, 1939, pp. 5-48 (in Russian).

[11] S.N. Kojevnikov, Foundation of structural synthesis of mechanisms, Kiyev, 1979, p. 229 (in Russian).

[12] O.G. Ozol, New structural formula for mechanisms and its theoretical and practical meaning, Latvia Agric. Acad. 11 (1962) 113-129 (in Russian).

[13] F. Freudenstein, The basic concepts of Polya's theory of enumeration, with application to the structural classification of mechanisms, J. Mech. 3 (1967) 275-290. 
[14] L. Dobrjanskyi, F. Freudenstein, Some applications of graph theory to the structural analysis of mechanisms, Trans. ASME J. Eng. Ind. (1967) 153-158.

[15] T.H. Davies, F.R.E. Crossley, Structural analysis of plane linkages by Franke's condensed notation, J. Mech. 1 (1966) 171-183.

[16] W.J. Sohn, F. Freudenstein, An application of dual graphs to the automatic generation of kinematic structures of mechanisms, Trans. ASME 108 (1986) 392-398.

[17] W.M. Hwang, Y.W. Hwang, Computer-aided structural synthesis of planar kinematic chains with simple joints, Mech. Mach. Theory 27 (2) (1992) 189-199.

[18] C.R. Tischler, A.E. Samuel, K.H. Hunt, Kinematic chains for robot hands-I orderly number synthesis, Mech. Mach. Theory 30 (1995) 1193-1215.

[19] A.C. Rao, P.B. Deshmukh, Computer aided structural synthesis of planar kinematic chains obviating the test for isomorphism, Mech. Mach. Theory 36 (2001) 489-506.

[20] D. Stewart, A platform with six degree of freedom, Proceedings of the Institution of Mechanical Engineers, part I 180 (5) (1956) 371-386.

[21] R.I. Alizade, E.T. Hajiyev, G.N. Sandor, Type synthesis of spatial mechanisms on the basis of spatial single loop structural groups, Mech. Mach. Theory 20 (2) (1985) 95-101.

[22] R.I. Alizade, Investigation of linkage mechanisms with lower pairs from point of view of its structure and classification, Azerb. Poly. Inst., Baku, 1988, pp. 111-127 (in Russian).

[23] R.I. Alizade, N.R. Tagiyev, J. Duffy, A forward and reverse displacement analysis of an in-parallel spherical manipulator, Mech. Mach. Theory 29 (1) (1994) 125-137.

[24] T. Dostalizade, On the dynamics of parallel manipulators, Ph.D. Dissertation, Azerb. Tech. Univ., Baku, 2004 (in Russian). 